\title{
1 Combinatorial engineering for photoautotrophic production of 2 recombinant products from the green microalga Chlamydomonas \\ 3 reinhardtii
}

4 Malak N. Abdallah", Gordon B. Wellman ${ }^{1}$, Sebastian Overmans ${ }^{1}$, Kyle J. Lauersen ${ }^{*, 1}$

5

$6{ }^{1}$ Bioengineering Program, Biological and Environmental Sciences and Engineering Division,

7 King Abdullah University of Science and Technology (KAUST), Thuwal 23955-6900,

8 Kingdom of Saudi Arabia

9

10 * Corresponding author: kyle.lauersen@kaust.edu.sa

14 Keywords: Microalgae, Phosphite, Algal Biotechnology, Waste Reuse, Metabolic

15 Engineering, Isoprenoids, Terpenoids 


\begin{abstract}
16 Abstract
17 Chlamydomonas reinhardtii has emerged as a powerful green cell factory for 18 metabolic engineering of sustainable products created from the photosynthetic 19 lifestyle of this microalga. Advances in nuclear genome and transgene expression 20 engineering are allowing robust engineering strategies to be demonstrated in this 21 host. However, commonly used lab strains are not equipped with features to enable 22 their broader implementation in non-sterile conditions and high-cell density concepts. 23 Here, we use combinatorial chloroplast and nuclear genome engineering to 24 complement the $C$. reinhardtii strain UVM4 with publicly available genetic tools to 25 enable the use of inorganic phosphite and nitrate as a sole the source phosphorous 26 and nitrogen, respectively. We present recipes to create phosphite-buffered media 27 solutions that enable high cell density algal cultivation. We then combine previously 28 reported engineering strategies to produce the heterologous sesquiterpenoid 29 patchoulol to high titers from our engineered green cell factories and show these 30 products are possible to produce in under non-sterile conditions. Our work presents 31 a straightforward means to generate $C$. reinhardtii strains for broader application in 32 bio-processes for the sustainable generation of products.
\end{abstract}


33

34

35

36

37

38

39

40

41

42

43

44

45

46

47

48

49

50

51

52

53

54

55

56

57

58

59

60

61

62

63

64

65

66

67

68

69

70

71

72

73

74

75

76

77

78

79

80

81

82

\section{Introduction}

The model green microalga Chlamydomonas reinhardtii has emerged in recent years as a newcomer in the metabolic engineering space due to enabling advances in transgene design (Baier et al., 2018b, 2020) and the use of nuclear mutants with enhanced transgene expression rates (Neupert et al., 2009). C. reinhardtii has been extensively used as a host for chloroplast genome engineering for the expression of recombinant proteins for several years (Wannathong et al., 2016; Dyo and Purton, 2018). However, this alga has historically demonstrated recalcitrance to nuclear transgene expression owing to genetic architectures with high guanine-cytosine (GC) nucleotide content and intron density, as well as a recently characterized epigenetic silencing mechanism (Neupert et al., 2020). Through a series of mutational events, strains UVM4 and UVM11 were generated which exhibited improvements in transgene expression over others (Neupert et al., 2009; Barahimipour et al., 2016). UVM4 has become a workhorse strain for demonstrations of efficient transgene expression, with examples of heterologous production of sesquiterpenes (Lauersen et al., 2016; Wichmann et al., 2018), diterpenes (Lauersen et al., 2018; Einhaus et al., 2021), and polyamines (Freudenberg et al., 2021), modified fatty acid and alkene contents (Yunus et al., 2018), secreted recombinant proteins (Lauersen et al., 2013b, 2013a, 2015a; Baier et al., 2018a), and altered pigment composition (Perozeni et al., 2020). The reduced epigenetic silencing of this strain coupled with improvements of synthetic intron-addition transgene design strategies (Baier et al., 2018b, 2020) and optimized regulatory element combinations (Scranton et al., 2016; Einhaus et al., 2021), have resulted in an increased momentum for algal synthetic biology and green biotechnology applications with these hosts (Lauersen, 2019). C. reinhardtii represents a model green microalga that has very well developed molecular toolkits, including optimized (Lauersen et al., 2015b; Wichmann et al., 2018) and modular cloning (MoClo) plasmids (Crozet et al., 2018). Although a great deal of advancement has been made in gene expression and genetic engineering design, major limitations to scalable cultivation of $C$. reinhardtii remain which limits the broader development of engineered algal bio-processes.

Cultivation of $C$. reinhardtii is conducted at neutral $\mathrm{pH}$, which means that the proteinrich algal cells are subject to rapid contamination/predation in non-sterile conditions. Sterility is difficult to maintain in large-scale cultivation concepts or in complicated bio-processes. Other industrially cultivated algae have features such as extreme $\mathrm{pH}$ or salinity tolerance which allow cultivation in selective conditions, or are dominant fast-growing, aggressive species. Although fast-growing, the UVM4 strain is also nitrate incapable. The use of nitrate is common in larger-scale algal cultivation media, as this nitrogen source does not cause significant $\mathrm{pH}$ shifts during its consumption. Complementation of nitrate metabolism is also important for increasing cell densities in cultivations, as has been recently demonstrated for the production of polyamines from this host (Freudenberg et al., 2021). The risk of contamination in addition to lack of nitrate metabolism capabilities make UVM4 deficient in features which would enable its broader use as an engineered algal green-cell factory outside of proof-of-principle laboratory experiments. One strategy for reducing contamination of algal cultures is the introduction of metabolic capacity for metabolism of inorganic phosphite as a phosphorous source. This has been shown in numerous organisms, including $C$. reinhardtii, to reduce contamination and act as a selection agent (LópezArredondo and Herrera-Estrella, 2012; Loera-Quezada et al., 2016; Changko et al., 2020; Cutolo et al., 2020; Dahlin and Guarnieri, 2022). Engineered expression of Pseudomonas stutzeri WM88 phosphite NAD+ oxidoreductase ptxD from either the 
83 chloroplast or nuclear genomes of algae has been shown to confer the ability to 84 metabolize phosphite (López-Arredondo and Herrera-Estrella, 2012; Changko et al., 85 2020; Cutolo et al., 2020; Dahlin and Guarnieri, 2022).

86 To date, demonstrated advances in nuclear transgene expression for metabolic 87 engineering described above have not incorporated combinatorial engineering with 88 chloroplast expression constructs in the same strain. Here, we combined published 89 advances in chloroplast engineering (Changko et al., 2020) with multiple nuclear 90 engineering steps in UVM4 to demonstrate growth of this strain in phosphite- and 91 nitrate-containing media while producing a proof-of-concept heterologous 92 sesquiterpenoid. We present recipes to enable high-cell density cultivation in 93 phosphite-buffered media and show that heterologous metabolites can be generated 94 in the presence of microbial contamination. Our work shows that advances in nuclear 95 and chloroplast engineering can be combined to yield more powerful green cell 96 chassis that are amenable to bio-process goals.

97

98 


\section{2. Materials and Methods}

\subsection{Algal cultivation and growth measurements}

101

Chlamydomonas reinhardtii strain UVM4 was used as the parental strain for

102 transformations. This strain was derived from several rounds of mutation in the parental CC-4350 by Dr. Juliane Neupert in the lab of Prof. Dr. Ralph Bock (Neupert et al., 2009) and contains a mutation in Sir2-type histone deacetylase (SRTA) which enables improved transgene expression rates from the algal nuclear genome (Neupert et al., 2020). UVM4 is not able to use nitrate as a nitrogen source due to nit1/nit2 locus mutations (Freudenberg et al., 2021). Microalgal cultures were routinely maintained in Tris acetate phosphate (TAP) medium (Gorman and Levine, 1965) with updated trace element solution (Kropat et al., 2011) and maintained under $150 \mu \mathrm{mol} \mathrm{m}^{-2} \mathrm{~s}^{-1}$ mixed cold and warm LED lights with 120-190 rpm agitation in shake flasks or microtiter plates. Light intensities and spectra were measured with a handheld spectrometer (Spectromaster C-7000, Sekonic). Ammonium in TAP salts solution was replaced with equimolar $\mathrm{NaNO}_{3}$ to make TAP-NO phosphate with phosphite to make TAPhi and $\mathrm{TAPhi}^{-\mathrm{NO}_{3}}$ media are described in Supplemental File 1. High-density 6xP medium was prepared as described in (Freudenberg et al., 2021) and buffered phosphite solutions to match molar concentrations of phosphorous to make 6xPhi medium are as described in Supplemental File 1. All phosphite solutions were filter sterilized and added to media after autoclaving. Cultivation in CellDeg HD100 cultivators (CellDeg GmbH, Germany) was performed with the indicated light and $\mathrm{CO}_{2}$ regimes by the growth control unit using either 6xP or 6xPhi media. Illumination was delivered by a Valoya broad spectrum LED board supplied by CellDeg GmbH (Germany, spectrum presented in Supplemental File 2).

Growth of algae and contaminants was analyzed by flow cytometry using an Invitrogen Attune NxT flow cytometer (Thermo Fisher Scientific, UK) equipped with a $488 \mathrm{~nm}$ blue laser for forward scatter and side scatter measurements, and a 695/40 $\mathrm{nm}$ filter to detect chlorophyll and non-fluorescent particles, respectively. All culture samples were diluted $1 / 100$ with $0.9 \% \mathrm{NaCl}$ solution and measured in technical triplicates using previously described settings (Overmans and Lauersen, 2022).

\subsection{Plasmids, algal transformation, and screening for phosphite and nitrate metabolism}

Plasmids used in this study are listed in Supplemental Table 1. All cloning and plasmid linearization was performed with Thermofisher FastDigest restriction enzymes, New England Biolabs Quick Ligase, and Q5 polymerase following manufacturer's protocols. Plasmids were maintained in chemically competent Escherichia coli DH5a transformed by heat shock. Glass bead transformation of $C$. reinhardtii was performed as previously described for both chloroplast and nuclear targeted genetic constructs (Kindle, 1990; Kindle et al., 1991). Chloroplast transformation of the $\mathrm{pPO}_{3}$ plasmid ((Changko et al., 2020) graciously provided by Prof. Saul Purton) was performed with $10 \mu \mathrm{g}$ circular DNA and $0.1 \mathrm{~mm}$ diameter glass beads rather than $0.424-0.600 \mathrm{~mm}$ as commonly used for nuclear transformation. Recovery was performed in $45 \mathrm{~mL}$ TAPhi liquid for $5 \mathrm{~d}$ with $150 \mu \mathrm{E}$ PAR prior to plating. Selection was achieved by plating on TAPhi agar plates incubated at $200 \mu \mathrm{E}$ for 2-3 weeks. Colonies were then grown in TAPhi liquid in microtiter plates until green. 
147 One transformant with clear growth in liquid TAPhi, hereafter named UVM4-phi, was

148 transformed for complementation of nitrate metabolic capacity by co-transformation

149 of linearized pMN24 (Fernández et al., 1989b) and pMN68 (Schnell and Lefebvre,

150 1993) (Chlamydomonas Resource Center, https://www.chlamycollection.org) by

151 glass beads as previously described (Freudenberg et al., 2021) with overnight

152

153

154

155

156

157

158

159

160

161

162

163

164

165

166

167

168

169

170 recovery and subsequent selection on $\mathrm{TAPhi}^{-\mathrm{NO}_{3}}$ agar plates. Transformant colonies recovered on $\mathrm{TAPhi}^{-\mathrm{NO}_{3}}$ plates were then compared in liquid media in 24well microtiter plates with standard lighting conditions at $180 \mathrm{rpm}$. Homoplasmy of the pPO3 integration into the chloroplast genome was determined by PCR using primers Fw: AATTGTATGGGCTCACAACAAACTTAAAGT and Rv: TAAAATTGTGAGACCATGAGTAATGTTCCTCC. The resulting transformants were also screened by iodine vapour assay as previously described (Wichmann et al., 2018) to determine if random integration had caused starch synthesis modifications. Modified UVM4 transformants which grew with phosphite and nitrate are referred to as UVM4-Phosphite-Nitrate (UPN) strains.

Efficiency of nuclear transgene expression of intermediate strains was investigated by glass bead transformation of the pOpt2_mVenus_Paro plasmid (Wichmann et al., 2018) followed by selection on each respective modified medium with $10 \mathrm{mg} \mathrm{L}^{-1}$ paromomycin and fluorescent reporter expression analysis. Fluorescent mVenus expression intensities were analyzed by picking primary transformant colonies using a PIXL robot (Singer Instruments, UK) to 384 colonies/plate layout on manufacturer supplied rectangular Petri dishes. After one week, colonies were replicated using the Singer Instruments ROTOR to generate imaging-ready colonies. White-light pictures of algae colony plates were taken in the built-in PIXL camera. Chlorophyll and mVenus fluorescence signals were captured in an Analytik Jena Chemstudio Plus gel doc with eLite halogen light source and excitation filters. Chlorophyll fluorescence was captured by $475 / 20 \mathrm{~nm}$ excitation with orange DNA gel emission filter with $1 \mathrm{sec}$ exposure, while mVenus signal was captured with 510/10 nm excitation and 530/10 $\mathrm{nm}$ emission filter with $30 \mathrm{sec}$ exposure.

\subsection{Generation of patchoulol producing UPN transformants}

Plasmids for algal nuclear genome-based expression of the Pogostemon cablin patchoulol synthase (PCPS, UniProt Q49SP3) were adapted from (Lauersen et al., 2016). The gene expression cassette for PCPS expression was modified from the pOpt2 vector concept of (Wichmann et al., 2018) to contain transgene designs presented in (Einhaus et al., 2021; Freudenberg et al., 2021). Briefly, PcPS expression here was driven by the hybrid heat shock 70A-beta-bubulin promoter described by (Einhaus et al., 2021) and the mVenus cassette was modified to contain 2 copies of the $C$. reinhardtii ribulose-1,5-bisphosphate carboxylase/oxygenase small subunit (RBCS2) intron 1. The RBCS2 intron 2 was moved into the C-terminal strep-II tag of the gene-of-interest expression cassette in the pOpt2_mVenus_Paro plasmid which confers paromomycin resistance in $C$. reinhardtii (Wichmann et al., 2018). PcPS was subcloned into BamHI-Bglll, and 2X, $3 \mathrm{X}$, and 4X PCPS expression cassettes were built by Scal-Bgll inserts from the previous plasmid subcloned into Scal-BamHI of the progenitor plasmid described in Supplemental Figure 1. All constructs contain the C-terminal mVenus (YFP) fusion which enabled plate-level fluorescence detection in UPN colonies picked by the PIXL robot. C. reinhardtii squalene synthase (UniProt A8IE29) knockdown was achieved by secondary transformation using the previously described pOpt2_cCA-gLuc_i3SQS_Spect plasmid (Wichmann et al., 2018). UPN PCPS-YFP + SQS k.d. double 
197 transformants were selected on $\mathrm{TAPhi}^{-\mathrm{NO}_{3}}$ agar media containing $10 \mathrm{mg} \mathrm{L}^{-1}$ 198 paromomycin and $200 \mathrm{mg} \mathrm{L}^{-1}$ spectinomycin as previously described (Wichmann et 199 al., 2018). YFP and luciferase signals of UPN colonies were captured in the 200 Chemstudio PLUS with previously described buffers and reagents for Gaussia 201 princeps luciferase bioluminescence analysis (Lauersen et al., 2013a). Full-length 202 target recombinant protein was determined by SDS PAGE and in-gel fluorescence of 203 whole cell pellets in the Chemstudio PLUS. All plasmid sequence files used in this 204 205 work are given in Supplemental File 3.

\subsubsection{Gas Chromatography analysis of patchoulol productivity}

UPN transformants expressing PCPS variants were screened for heterologous patchoulol productivity by cultivation in $4.5 \mathrm{~mL}$ TAPhi- $\mathrm{NO}_{3}$ media with $500 \mu \mathrm{l}$ dodecane overlay in triplicates for $6 \mathrm{~d}$ as previously described (Lauersen et al., 2018). Six individual transformants were investigated for each plasmid construct or combination after fluorescence, or fluorescence and luciferase, screening at the agar-plate level. Dodecane samples were collected from cultures, $90 \mu \mathrm{l}$ of each collected dodecane sample was transferred into triplicate GC vials. A patchoulol standard (18450, Cayman Chemical Company, USA) calibration curve in the range 10-200 $\mu \mathrm{M}$ patchoulol in dodecane was used for linear-range quantification. $250 \mu \mathrm{M}$ of alpha-humulene (CRM40921, Sigma-Aldrich, USA) was added as an internal standard to each dodecane sample and patchoulol standard. Quantification methods and calculations are shown in Supplemental File 4. The dodecane samples were analyzed using an Agilent 7890A gas chromatograph (GC) equipped with a DB-5MS column (Agilent J\&W, USA) attached to a 5975C mass spectrometer (MS) with tripleaxis detector (Agilent Technologies, USA). A previously described GC oven temperature protocol was used (Overmans and Lauersen, 2022). All GC-MS measurements were performed in triplicate $(n=3)$, and chromatograms were manually reviewed for quality control. Gas chromatograms were evaluated with MassHunter Workstation software version B.08.00 (Agilent Technologies, USA).

\subsection{Test of intentional contamination in cultures}

To test the ability of engineered $C$. reinhardtii UPN lines to withstand contamination in non-sterile conditions using media modifications presented in this work, cultivation

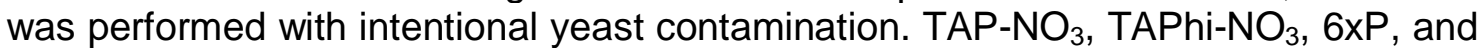
6xPhi media were used to cultivate an engineered SQS k.d. + 2XPCPs expressing UPN transformant. Saccharomyces cerevisiae (yeast) cells were cultured in Yeast Extract-Peptone-Dextrose (YPD) medium (Cold Spring Harbor Protocols) overnight at $28^{\circ} \mathrm{C}$. The following day, pelleted cells were resuspended in $300 \mathrm{~mL} 6 \mathrm{xP}$ or $6 x \mathrm{Phi}$ media. Triplicate wells in 6-well microtiter plates containing $4 \mathrm{~mL}$ dilute UPN patchoulol culture in 6xP or 6xPhi media were inoculated with either $500 \mu$ of yeastsolutions (above) or clean medium as controls. $500 \mu \mathrm{l}$ of $\mathrm{n}$-dodecane overlay was also added to each well. Approximately $3 \mathrm{~mL}$ of concentrated potassium bicarbonate buffer was added between the wells to provide a dilute $\mathrm{CO}_{2}$ atmosphere as previously described (Dienst et al., 2020). Cultures in TAP-media were grown for $6 \mathrm{~d}$ and $6 x \mathrm{P} / \mathrm{Phi}$ for $9 \mathrm{~d}$ on laboratory shakers at $120 \mathrm{rpm}$ with $12 \mathrm{~h}: 12 \mathrm{~h}$ light:dark cycle $(150 \mu \mathrm{E})$. Cell densities and patchoulol productivities were analyzed as described above.

244

245

246 
247

248

249

250

251

252

253

254

255

256

257

258

259

260

261

262

263

264

265

266

267

268

269

270

271

272

273

274

275

276

277

278

279

280

281

282

283

284

285

286

287

288

289

290

291

292

293

294

295

\section{Results}

\subsection{Phosphite and nitrate metabolism can be combined in the nuclear mutant UVM4}

The C. reinhardtii mutants UVM4 and UVM11(Neupert et al., 2009) exhibit reduced transgene silencing due to a mutation in the in Sir2-type histone deacetylase (SRTA) (Neupert et al., 2020). UVM4 has served as a powerful parent strain for many recent examples of metabolic engineering in this green microalga (Lauersen et al., 2016, 2018; Wichmann et al., 2018; Einhaus et al., 2021; Freudenberg et al., 2021). Despite its value for past experiments, the alga is grown at neutral $\mathrm{pH}$ and contains mutations in its nitrate metabolism which prevent use of this nitrogen source. These two features manifest in high-risk of microbial contamination and the inability to use industrially relevant culture media (Changko et al., 2020; Freudenberg et al., 2021). To prepare UVM4 strains for broader applications, we set to complement it with the capacity to use phosphite as a P source and nitrate as an N source.

We complemented UVM4 with plasmid pPO3 (Changko et al., 2020), to express the $P$. stutzeri WM88 phosphite NAD+ oxidoreductase ptxD (López-Arredondo and Herrera-Estrella, 2012) that converts inorganic phosphite into organic phosphate from the algal chloroplast genome (Figure 1A). We found it was possible to transform UVM4 with this chloroplast genome-integrating plasmid by glass bead transformation and select colonies on TAPhi medium with no additional selection pressure. A resulting UVM4-phi transformant was then subsequently transformed with pMN24 and pMN68 plasmids which contain genomic copies of the nit1 and nit2 loci respectively to complement nitrate metabolism capacity (Fernández et al., 1989a; Schnell and Lefebvre, 1993) (Figure 1A).

Colonies were recovered by selection on $\mathrm{TAPhi}^{-\mathrm{NO}_{3}}$ plates with no antibiotic (Figure 1A). Complementation with nit $1 / 2$ can sometimes lead to colonies that survive on agar plate, but do not perform well in liquid medium. Therefore, we also benchmarked performance of 24 UVM4-phosphite-nitrate (UPN) colonies derived from these transformations in $\mathrm{TAPhi}-\mathrm{NO}_{3}$ and photoautotrophic cultivation with $\mathrm{CO}_{2}$ as a carbon source (Figure 1A, lower right). Parental strains were not able to grow in the selective media for each plasmid. UPN strains grown in liquid medium exhibited variable performance, especially in photoautotrophic conditions (Figure 1A,B). Most colonies maintained normal starch accumulation, which was qualitatively assessed by iodine vapour, however, colonies 19 and 23 showed reduced iodine staining (Figure 1C). Homoplasmy of pPO3 integration was determined in UVM4-phi and nitrate complemented strains (Supplemental Figure 2).

To confirm that the three plasmid integrations did not modify the performance of the parent UVM4 capacities for nuclear transgene expression, several UPN strains with acceptable growth in liquid phosphite-nitrate media were transformed with a YFP reporter plasmid(Wichmann et al., 2018). High-throughput robotics assisted colony picking allowed analysis of between 943-1079 colonies for each strain and platelevel reporter imaging was used to quantify reporter expression across the populations. YFP reporter expression efficiencies in the final UPN strains were comparable to parent UVM4 (Figure 1D).

\subsection{Phosphite can replace phosphate in buffered media for high cell-density cultivation of algal cells \\ Using mono- and di-basic forms of phosphite, we generated a buffered phosphite solution to emulate the phosphate buffer solution of recently published 6xP medium}


296 (Freudenberg et al., 2021) (Supplemental File 1). In order to compare whether our 297 high-density phosphite medium (6xPhi) could be used in a comparable fashion to 298 6xP, we benchmarked growth of a UPN strain in a high-density cultivation concept 299 using high-light and membrane delivered $\mathrm{CO}_{2}$ in CellDeg HD100 cultivators (Figure

300 2A). We did not observe differences in performance for the UPN strain cultivated in $3016 x \mathrm{P}$ or $6 \mathrm{xPhi}$ which reached comparable cell densities throughout cultivation (Figure 302 2B).

303

304

305

306

307

308

309

310

311

312

313

314

\subsection{Heterologous products can be efficiently made in UPN strains}

We then chose to combine two proven engineering strategies for sesquiterpenoid

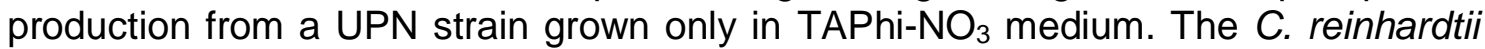
codon optimized P. cablin patchoulol synthase (PCPS) was expressed in 1, 2, 3, and $4 \mathrm{X}$ copy fusion protein constructs with C-terminal YFP from the nuclear genome of this alga (Figure 3). Robotics assisted colony picking and YFP screening allowed selection of six transformants per plasmid with confirmed PCPS expression which were benchmarked for patchoulol productivity as previously described (Lauersen et al., 2018). The best performing transformants were subsequently transformed with a secreted luciferase-artificial-micro-RNA expression construct targeting the $C$. reinhardtii squalene synthase (SQS)(Wichmann et al., 2018). After colony recovery and robotics picking, plate-level imaging was used to isolate colonies with YFP fluorescence signal (PCPS) and luciferase activity (SQS k.d.) (Figure 3). Patchoulol productivity analysis indicated striking improvements in patchoulol productivity for SQS k.d. strains compared to parentals with the best performing strains generating $\sim 145 \mathrm{fg}$ patchoulol cell ${ }^{-1}$ (Figure 3). Full-length fusion protein expression could be confirmed only for 1-3X PCPS-YFP constructs by in gel fluorescence (Supplemental Figure 3).

\subsection{Nitrate and phosphite can both assist contaminant control in algal cultures}

As contamination of cultures can be an issue with neutral $\mathrm{pH}$ cultivation, we wanted to determine if phosphite and nitrate could permit algal growth in the presence of contamination. We intentionally contaminated the best performing UPN PCPS SQS k.d. strain in mixotrophic (acetic acid) and photoautotrophic cultures in media with nitrate as a nitrogen source and either phosphate or phosphite as a phosphorous source. Yeast cells were added to cultures directly in higher cellular abundances than algal cells (Figure 4). In all media conditions, yeast cells did not proliferate, regardless of the presence of organic carbon, but also did not reduce in number. When acetic acid (TAP-derived) media were used, algal growth was reduced compared to cultivations without yeast, also with phosphite (Figure 4). No difference in performance was noted in photoautotrophic cultures. In all conditions, the presence of high concentrations of yeasts in cultivations did not inhibit heterologous patchoulol production (Figure 4).

We then benchmarked patchoulol productivity in a $200 \mathrm{~mL}$ culture in a membrane gas delivery bioreactor containing $10 \%$ dodecane overlay to capture heterologous sesquiterpenoid product. Culture volume was adjusted to $200 \mathrm{~mL}$ to avoid contact of dodecane with the hydrophobic gas delivery membrane during shaking and the culture was operated in non-sterile conditions. The culture accumulated up to $6.5 \times 10^{7} \pm 1.9 \times 10^{6}$ cells $\mathrm{mL}^{-1}$ and generated $6.2 \mathrm{mg} \mathrm{L}^{-1}$ patchoulol in 6 days using this system. 


\section{4. Discussion}

347 4.1. Designing engineerable strains to be ready for bio-processes

348 We chose phosphite metabolism complementation with plasmid pPO3 as this also 349 contains extra future chloroplast genome engineering potential through the addition of the W $W_{\text {TGA }}$ tRNA for tryptophan as previously described (Changko et al., 2020). When filter sterilization was used, transformation and selection on phosphite solutions was greatly improved and appearance of background algal growth at the plate level was reduced (data not shown). After confirmed growth of pPO3 transformants in liquid phosphite, nitrate metabolism was complemented by transformation of both pMN24 (NIT1) and pMN68 (NIT2) plasmids in UVM4. This double transformation is relatively inefficient, nevertheless, we could generate several dozen colonies per transformation which recovered on nitrate plates. Colonies which recovered on nitrate plates did, however, not all perform well in liquid culture growth in nitrate-containing liquid media. We chose to move forward with only those colonies which appeared to grow to dark green stationary phase (Figure 1A). Colonies were then checked for homoplasmy integration of the pPO3 phosphite conferring plasmid (Supplemental Figure 2) and two were benchmarked for their growth in phosphite- and nitrate-containing media compared to their parental strains (Figure 1B).

To determine if our strategy for UVM4 augmentation would allow future engineering to benefit from these metabolic enhancements, two questions remained: 1.) was nuclear transformation expression efficiency disturbed during these events in UVM4 derivatives? 2.) Can inorganic phosphite be used in a similar way to organic phosphate, for buffered media solutions? We benchmarked two fully complemented UPN transformants, their UVM4-phi parent, and the UVM4 starting strain for YFP efficiency expression from the nuclear genome. Using high-throughput colony picking, we were able to analyze $\sim 1000$ colonies per transformation event and compare YFP expression efficiencies across the populations (Figure 1C). Although some variance, there was little difference could be seen in the total ratio of high and mid-range YFP expressing colonies, suggesting our three plasmid integrations had not modified this capacity.

We then set out to make a buffered phosphite solution which could replace buffered phosphate solutions in culture media (Supplemental File 1). In direct comparisons of growth tests, the UPN strain did not show performance differences in phosphite compared to phosphate in photoautotrophic high-density cultivations (Figure 2). Our results indicate this is an effective strategy to use inorganic phosphite as a media component. As phosphate is a globally dwindling resource important to agriculture, bio-conversion of phosphite into phosphate may also enable the use of this waste mineral to yield bio-fertilizers through algal cultivation.

\subsection{Patchoulol production in metabolically complemented strains}

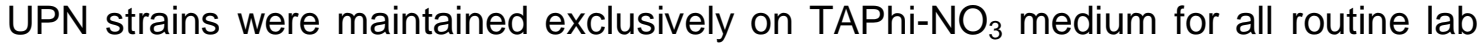
work. A further aim was to determine if it was possible to conduct additional metabolic engineering in these strains for heterologous isoprenoid production using $\mathrm{Phi}-\mathrm{NO}_{3}$ media. Plasmids were constructed based on previous designs to express the patchoulol synthase (PCPS) and localize it in the cytoplasm of the alga where this enzyme is known to convert freely available farnesyl pyrophosphate (FPP) into patchouli alcohol (patchoulol)(Lauersen et al., 2016). We chose to combine recently published modifications in promoter(Einhaus et al., 2021) and intron 
395 use(Freudenberg et al., 2021) (Supplemental Figure 1), in order to assist 396 recombinant protein accumulation in an effort to enhance product yields. Previous work on PCPS, indicated cellular patchoulol productivities could be enhanced when the protein was fused to itself in a repetitive fashion to yield more active sites per translated protein(Lauersen et al., 2016). Here, we copied this gene design strategy and combined it with an artificial micro-RNA (amiRNA) knockdown of the squalene synthase (SQS) (Figure 3). It was previously found that SQS k.d. improved (E)- $\alpha$ -

401

402

403

404

405 biabolene titers from the cytoplasm of $C$. reinhardtii as this is the direct competitor for FPP precursor(Wichmann et al., 2018). Additive PcPS units were found here to increase cellular patchoulol yields as previously observed (Lauersen et al., 2016). However, increasing repetitions past 3 PCPS copies was found to be unstable and

406

407

408

409

410

411

412

413

414

415

416

417 did not generate reliable patchoulol expression strains (Figure 3, Supplemental Figure 3). As expected based on past work with bisabolene(Wichmann et al., 2018), addition of the SQS k.d. to the best performing PCPS variant of each plasmid lead to transformants with drastic improvements in patchoulol productivity. Previous engineering of patchoulol production for $C$. reinhardtii lead to a maximal volumetric productivity of $\sim 350 \mu \mathrm{g}$ patchoulol $\mathrm{L}^{-1}$ in mixotrophic $400 \mathrm{~mL}$ bioreactor conditions from a 3XPCPS-YFP transformant(Lauersen et al., 2016). Here, a 2XPcPS transformant subsequently transformed with the SQS k.d. generated lines producing 700-1400 $\mu \mathrm{g}$ patchoulol $\mathrm{L}^{-1}$ culture (Figure 3, Supplemental Figures 4 and 5). Apparent improvements in 1-4X PCPS-YFP lines were observed by SQS k.d. and from 1-3XPCPS-YFP, mean production increased with increasing PcPS units from $1 \mathrm{X}-3 \mathrm{XPCPS}$. Maximal cellular productivity was observed in a single 2XPCPS SQS k.d. line, with up to $143 \mathrm{fg}$ patchoulol cell ${ }^{-1}$ (Figure 3).

420

421

422

423

424

425

426

427

428

429

430

431

432

433

434

435

436

437

438

439

A risk to scaled cultivation of engineered $C$. reinhardtii in bio-production concepts is contamination and reduced productivities, which is especially true for cell-wall deficient strains that may be more readily outcompeted by contaminants. We intentionally contaminated mixotrophic and photoautotrophic media containing either $\mathrm{NO}_{3}$ or Phi and $\mathrm{NO}_{3}$ with yeast, and cultivated a UPN-patchoulol producing strain in these sub-optimal conditions (Figure 4A). We inoculated the 2XPCPS-SQS k.d. UPN strain into media containing $6.0 \times 10^{6}$ cells $\mathrm{mL}^{-1}$ yeast, the same cell density as reached in mid-log phase for the algal cells. We chose to provide $\mathrm{CO}_{2}$ using potassium bicarbonate buffers(Dienst et al., 2020) rather than direct gas delivery to further challenge the phototrophic cultures. In all conditions, yeast cells did not proliferate, with either acetic acid as a carbon source, or in the photoautotrophic media (Figure 4A). Mixotrophic cultures exhibited lower algal cell densities in later stages of cultivation than in the absence of yeast, likely due to the yeast sequestering some of the acetic acid. However, in the establishment phase (days 0 3 ), growth with yeast was not markedly different than that of cultures without yeast. In photoautotrophic cultures, yeast was inoculated at higher starting cell densities $\left(8 \times 10^{6}\right.$ cells $\left.\mathrm{mL}^{-1}\right)$ as there is no organic carbon source. This density was chosen to determine if the yeast cells would competitively inhibit the inoculated algal cells. Here, the UPN strain was able to overtake the yeast cells, demonstrating linear growth in both conditions relative to carbon diffusion rates in the medium. Under all conditions, the presence of yeast contaminants did not hinder the accumulation of heterologous patchoulol in dodecane overlays which exhibited similar productivities per algal cell (Figure 4A). Our results indicate that both nitrate and phosphite are a 441

442

443 powerful combination to limit contaminating microbial competitors in engineered algal cultivation concepts. 
444 To determine if we could produce patchoulol in non-sterile conditions, we cultivated

445 this strain in a CellDEG HD100 bioreactor with dodecane overlay using 6xPhi

446 medium (Figure 4B). Dodecane impairs the hydrophobic gas delivery membrane of

447 the reactors, so we used $200 \mathrm{~mL}$ culture volume to prevent the solvent interacting

448 with the membrane. Previous photoautotrophic yields of this product were only $449350 \mathrm{\mu g} \mathrm{L}^{-1}$ in 8 days (Lauersen et al., 2016). Here, without any process optimization, $450 \sim 6.2 \mathrm{mg}$ patchoulol $\mathrm{L}^{-1}$ was produced from $\mathrm{CO}_{2}$ in 7 days (Figure 4). A previous 451 study with Synechocystis sp. PCC 6803 used a similar membrane gas delivery 452 system for $10 \mathrm{~mL}$ cultures to generate up to $17.3 \mathrm{mg}$ patchoulol $\mathrm{L}^{-1}$ in 8 days using a 453 two-stage semi batch mode where half of the culture medium was replaced after 96 454 hours. We did not further optimize our production experiments in the HD100, as the 455 risk of dodecane-membrane wetting means cultivation must be performed with 456 volumes not intended for the system. A further issue of the dodecane overlay in 457 turbid algal cultures is the formation of emulsions with hydrophobic cellular 458 components and the dodecane solvent (Lauersen, 2019). The surface interaction of 459 culture and dodecane causes significant emulsion formation in this volume ratio 460 (Figure 4B), which is less pronounced in smaller volume cultivation units. 461 Nevertheless, our results indicated the combination of nitrate and phosphite 462 metabolic capacities enable high-density cultivations of engineered strains to be 463 performed with reduced risks of contamination without affecting process yields. To 464 our knowledge, this is the first demonstration of combinatorial plastid and nuclear 465 genome engineering in a green alga to generate a strain producing a heterologous 466 product.

467

\subsection{Conclusions}

Here, we have demonstrated the complementation of the common UVM4 nuclear mutant with the genetic capacity for phosphite and nitrate metabolism. These modifications were possible without affecting the nuclear transgene expression abilities of UVM4 and enabled the engineering of heterologous isoprenoid production in strains capable of growth in contamination reducing media. We present a recipe for buffered phosphite solutions to replace those of phosphate in common $C$. reinhardtii media and show improved titers of patchoulol though combination of strategies known to improve flux to sesquiterpenoid products. Our work can be used as a guide for others to adapt phosphite-nitrate metabolism into their strains and may enhance the transition of lab-scale engineering to less-sterile production concepts. 


\section{Acknowledgements}

482 Subcloning of PcPS plasmid 1X was performed in the lab of Prof. Dr. Olaf Kruse by 483 Dr. Julian Wichmann and Dr. Thomas Baier as part of an Institute for Innovation 484 Transfer (IIT), Universität Bielefeld, project funded by Lauersen (KAUST). The 485 authors are grateful to Saul Purton for providing plasmid pPO3 and Prof. Dr. Ralph 486 Bock for graciously providing strain UVM4 through MTA between the Max Planck 487 Institute of Molecular Physiology and KAUST. We would like to express thanks to 488 SSB group members for cooperation and collaboration during this project. The 489 research reported in this publication was supported by baseline funding from KAUST 490 to KL.

491 
492

493

494

495

496

497

498

499

500

501

502

503

504

505

506

507

508

509

510

511

512

513

514

515

516

517

518

519

520

521

522

523

524

525

526

527

528

529

530

531

532

533

534

535

536

537

538

539

540

541

\section{References}

Baier, T., Jacobebbinghaus, N., Einhaus, A., Lauersen, K. J., and Kruse, O. (2020). Introns mediate post-transcriptional enhancement of nuclear gene expression in the green microalga Chlamydomonas reinhardtii. PLOS Genet. 16, e1008944. doi:10.1371/journal.pgen.1008944.

Baier, T., Kros, D., Feiner, R. C., Lauersen, K. J., Müller, K. M., and Kruse, O. (2018a). Engineered Fusion Proteins for Efficient Protein Secretion and Purification of a Human Growth Factor from the Green Microalga Chlamydomonas reinhardtii. ACS Synth. Biol. 7, 2547-2557. doi:10.1021/acssynbio.8b00226.

Baier, T., Wichmann, J., Kruse, O., and Lauersen, K. J. (2018b). Intron-containing algal transgenes mediate efficient recombinant gene expression in the green microalga Chlamydomonas reinhardtii. Nucleic Acids Res. 46, 6909-6919. doi:10.1093/nar/gky532.

Barahimipour, R., Neupert, J., and Bock, R. (2016). Efficient expression of nuclear transgenes in the green alga Chlamydomonas: synthesis of an HIV antigen and development of a new selectable marker. Plant Mol. Biol., 1-16. doi:10.1007/s11103-015-0425-8.

Changko, S., Rajakumar, P. D., Young, R. E. B., and Purton, S. (2020). The phosphite oxidoreductase gene, ptxD as a bio-contained chloroplast marker and crop-protection tool for algal biotechnology using Chlamydomonas. Appl. Microbiol. Biotechnol. 104, 675-686. doi:10.1007/s00253-019-10258-7.

Crozet, P., Navarro, F. J., Willmund, F., Mehrshahi, P., Bakowski, K., Lauersen, K. J., et al. (2018). Birth of a Photosynthetic Chassis: A MoClo Toolkit Enabling Synthetic Biology in the Microalga Chlamydomonas reinhardtii. ACS Synth. Biol. 7, 2074-2086. doi:10.1021/acssynbio.8b00251.

Cutolo, E., Tosoni, M., Barera, S., Herrera-Estrella, L., Dall'Osto, L., and Bassi, R. (2020). A Phosphite Dehydrogenase Variant with Promiscuous Access to Nicotinamide Cofactor Pools Sustains Fast Phosphite-Dependent Growth of Transplastomic Chlamydomonas reinhardtii. Plants 9, 473. doi:10.3390/plants 9040473 .

Dahlin, L. R., and Guarnieri, M. T. (2022). Heterologous expression of phosphite dehydrogenase in the chloroplast or nucleus enables phosphite utilization and genetic selection in Picochlorum spp. Algal Res. 62, 102604. doi:10.1016/j.algal.2021.102604.

Dienst, D., Wichmann, J., Mantovani, O., Rodrigues, J. S., and Lindberg, P. (2020). High density cultivation for efficient sesquiterpenoid biosynthesis in Synechocystis sp. PCC 6803. Sci. Rep. 10, 5932. doi:10.1038/s41598-02062681-w.

Dyo, Y. M., and Purton, S. (2018). The algal chloroplast as a synthetic biology platform for production of therapeutic proteins. Microbiology, 1-9. doi:10.1099/mic.0.000599.

Einhaus, A., Baier, T., Rosenstengel, M., Freudenberg, R. A., and Kruse, O. (2021). Rational Promoter Engineering Enables Robust Terpene Production in Microalgae. ACS Synth. Biol. doi:10.1021/acssynbio.0c00632.

Fernández, E., Schnell, R., Ranum, L. P., Hussey, S. C., Silflow, C. D., and Lefebvre, P. A. (1989a). Isolation and characterization of the nitrate reductase structural gene of Chlamydomonas reinhardtii. Proc. Natl. Acad. Sci. U. S. A. 86, 6449-6453. doi:10.1073/pnas.86.17.6449.

Fernández, E., Schnell, R., Ranum, L. P., Hussey, S. C., Silflow, C. D., and 
542

543

544

545

546

547

548

549

550

551

552

553

554

555

556

557

558

559

560

561

562

563

564

565

566

567

568

569

570

571

572

573

574

575

576

577

578

579

580

581

582

583

584

585

586

587

588

589

590

591

Lefebvre, P. A. (1989b). Isolation and characterization of the nitrate reductase structural gene of Chlamydomonasreinhardtii. Proc. Natl. Acad. Sci. 86, 64496453. Available at: http://www.pnas.org/content/86/17/6449.abstract.

Freudenberg, R. A., Baier, T., Einhaus, A., Wobbe, L., and Kruse, O. (2021). High cell density cultivation enables efficient and sustainable recombinant polyamine production in the microalga Chlamydomonas reinhardtii. Bioresour. Technol. 323, 124542. doi:10.1016/j.biortech.2020.124542.

Gorman, D. S., and Levine, R. P. (1965). Cytochrome $f$ and plastocyanin: their sequence in the photosynthetic electron transport chain of Chlamydomonas reinhardi. Proc. Natl. Acad. Sci. 54, 1665-1669. doi:10.1073/pnas.54.6.1665.

Kindle, K. L. (1990). High-frequency nuclear transformation of Chlamydomonas reinhardtii. Proc. Natl. Acad. Sci. USA 87, 1228-1232. Available at: http://www.pnas.org/content/87/3/1228.abstract.

Kindle, K. L., Richards, K. L., and Stern, D. B. (1991). Engineering the chloroplast genome: Techniques and capabilities for chloroplast transformation in Chlamydomonas reinhardtii. Proc. Natl. Acad. Sci. U. S. A. 88, 1721-1725. doi:10.1073/pnas.88.5.1721.

Kropat, J., Hong-Hermesdorf, A., Casero, D., Ent, P., Castruita, M., Pellegrini, M., et al. (2011). A revised mineral nutrient supplement increases biomass and growth rate in Chlamydomonas reinhardtii. Plant J. 66, 770-780. doi:10.1111/j.1365313X.2011.04537.x.

Lauersen, K. J. (2019). Eukaryotic microalgae as hosts for light-driven heterologous isoprenoid production. Planta 249, 155-180. doi:10.1007/s00425-018-3048-x.

Lauersen, K. J., Baier, T., Wichmann, J., Wördenweber, R., Mussgnug, J. H., Hübner, W., et al. (2016). Efficient phototrophic production of a high-value sesquiterpenoid from the eukaryotic microalga Chlamydomonas reinhardtii. Metab. Eng. 38, 331-343. doi:10.1016/j.ymben.2016.07.013.

Lauersen, K. J., Berger, H., Mussgnug, J. H., and Kruse, O. (2013a). Efficient recombinant protein production and secretion from nuclear transgenes in Chlamydomonas reinhardtii. J. Biotechnol. 167, 101-110. doi:http://dx.doi.org/10.1016/j.jbiotec.2012.10.010.

Lauersen, K. J., Huber, I., Wichmann, J., Baier, T., Leiter, A., Gaukel, V., et al. (2015a). Investigating the dynamics of recombinant protein secretion from a microalgal host. J. Biotechnol. 215, 62-71. doi:10.1016/j.jbiotec.2015.05.001.

Lauersen, K. J., Kruse, O., and Mussgnug, J. H. (2015b). Targeted expression of nuclear transgenes in Chlamydomonas reinhardtii with a versatile, modular vector toolkit. Appl. Microbiol. Biotechnol. 99, 3491-3503. doi:10.1007/s00253014-6354-7.

Lauersen, K. J., Vanderveer, T. L., Berger, H., Kaluza, I., Mussgnug, J. H., Walker, V. K., et al. (2013b). Ice recrystallization inhibition mediated by a nuclearexpressed and -secreted recombinant ice-binding protein in the microalga Chlamydomonas reinhardtii. Appl. Microbiol. Biotechnol. 97, 9763-9772. doi:10.1007/s00253-013-5226-x.

Lauersen, K. J., Wichmann, J., Baier, T., Kampranis, S. C., Pateraki, I., Møller, B. L., et al. (2018). Phototrophic production of heterologous diterpenoids and a hydroxy-functionalized derivative from Chlamydomonas reinhardtii. Metab. Eng. 49, 116-127. doi:10.1016/j.ymben.2018.07.005.

Loera-Quezada, M. M., Leyva-González, M. A., Velázquez-Juárez, G., SanchezCalderón, L., Do Nascimento, M., López-Arredondo, D., et al. (2016). A novel genetic engineering platform for the effective management of biological 
592

593

594

595

596

597

598

599

600

601

602

603

604

605

606

607

608

609

610

611

612

613

614

615

616

617

618

619

620

621

622

623

624

625

626

627

628

629

630

631

632

633 contaminants for the production of microalgae. Plant Biotechnol. J. 14, 20662076. doi:10.1111/pbi.12564.

López-Arredondo, D. L., and Herrera-Estrella, L. (2012). Engineering phosphorus metabolism in plants to produce a dual fertilization and weed control system. Nat. Biotechnol. 30, 889-893. doi:10.1038/nbt.2346.

Neupert, J., Gallaher, S. D., Lu, Y., Strenkert, D., Barahimipour, R., Fitz-gibbon, S. T., et al. (2020). An epigenetic gene silencing pathway selectively acting on transgenic DNA in the green alga Chlamydomonas. Nat. Commun., 1-92. doi:10.1038/s41467-020-19983-4.

Neupert, J., Karcher, D., and Bock, R. (2009). Generation of Chlamydomonas strains that efficiently express nuclear transgenes. Plant J. 57, 1140-1150. doi:10.1111/j.1365-313X.2008.03746.x.

Overmans, S., and Lauersen, K. J. (2022). Biocompatible fluorocarbon liquid underlays for in situ extraction of isoprenoids from microbial cultures. bioRxiv. doi:10.1101/2022.01.27.477974.

Perozeni, F., Cazzaniga, S., Baier, T., Zanoni, F., Zoccatelli, G., Lauersen, K. J., et al. (2020). Turning a green alga red: engineering astaxanthin biosynthesis by intragenic pseudogene revival in Chlamydomonas reinhardtii. Plant Biotechnol. J. 18, 2053-2067. doi:10.1111/pbi.13364.

Schnell, R. A., and Lefebvre, P. A. (1993). Isolation of the chlamydomonas regulatory gene NIT2 by transposon tagging. Genetics 134, 737-747. doi:NIT21.

Scranton, M. A., Ostrand, J. T., Georgianna, D. R., Lofgren, S. M., Li, D., Ellis, R. C., et al. (2016). Synthetic promoters capable of driving robust nuclear gene expression in the green alga Chlamydomonas reinhardtii. Algal Res. 15, 135142. doi:10.1016/j.algal.2016.02.011.

Wannathong, T., Waterhouse, J. C., Young, R. E. B., Economou, C. K., and Purton, S. (2016). New tools for chloroplast genetic engineering allow the synthesis of human growth hormone in the green alga Chlamydomonas reinhardtii. Appl. Microbiol. Biotechnol. doi:10.1007/s00253-016-7354-6.

Wichmann, J., Baier, T., Wentnagel, E., Lauersen, K. J., and Kruse, O. (2018). Tailored carbon partitioning for phototrophic production of (E)-a-bisabolene from the green microalga Chlamydomonas reinhardtii. Metab. Eng. 45, 211-222. doi:10.1016/j.ymben.2017.12.010.

Yunus, I. S., Wichmann, J., Wördenweber, R., Lauersen, K. J., Kruse, O., and Jones, P. R. (2018). Synthetic metabolic pathways for photobiological conversion of $\mathrm{CO} 2$ into hydrocarbon fuel. Metab. Eng. 49, 201-211. doi:10.1016/j.ymben.2018.08.008.

Zabawinski, C., Koornhuyse, N. V. a N. D. E. N., Hulst, C. D., Schlichting, R., Giersch, C., Delrue, B., et al. (2001). Starchless Mutants of Chlamydomonas reinhardtii Lack the Small Subunit of a Heterotetrameric ADP-Glucose Pyrophosphorylase. 183, 1069-1077. doi:10.1128/JB.183.3.1069. 


\section{Figure legends}

635 Figure 1. Complementation of C. reinhardtii strain UVM4 for growth on phosphite

636 and nitrate. A Plasmid pPO3 (Changko et al., 2020) was transformed into UVM4 and

637 colonies were recovered on TAPhi agar medium. Colonies were then cultivated in

638 TAPhi liquid medium and one strain (UVM4-Phi) was selected for further

639 complementation with pMN24(Fernández et al., 1989b) and pMN68 (Schnell and

640 Lefebvre, 1993) (nit1/nit2) plasmids. Selection was performed on $\mathrm{TAPhi}^{-\mathrm{NO}_{3} \text { plates }}$

641 and resultant colonies capable of growth on phosphite and nitrate (UPN) were

642 cultivated in liquid mixotrophic (TAPhi-NO $\mathrm{NO}_{3}$ ) and autotrophic (6xPhi) media. Parental

643 strains were grown as reference in each previous stage media as shown. B Growth

644 curves of liquid cultures of selected colonies which performed well in $\mathrm{TAPhi}^{-\mathrm{NO}_{3}}$

645 media in TAPhi-NO $\mathrm{NO}_{3}$ or 6xPhi media. Parental strains UVM4 and UVM4-phi, were not

646 able to proliferate in the nitrate phosphite containing media. C UPN strains were

647 investigated by iodine vapour staining at the agar plate level to determine if the

648 transformation of three plasmids above had caused background mutations in starch

649 synthesis. Dark colour of colonies indicates presence of starch, yellow or light colour

650 indicates perturbed starch metabolism as shown for the starchless sta6 (Zabawinski

651 et al., 2001) mutant. Lighter starch staining is observed in UPN strains 19 and 23. D

652 Strains UVM4, UVM4-Phi, UPN1 and UPN22 were transformed with the

653 pOpt2_mVenus_Paro plasmid (cartoon) conferring paromomycin resistance and

654 expressing the mVenus (YFP reporter). High-throughput robotic colony picking and

655 fluorescence imaging was used to benchmark YFP expression across the

656 transfomant population. Chlorophyll fluorescence (red) was used to identify true

657 colonies, and YFP fluorescence (yellow) was graded for intensity of signal and

658 plotted comparing numbers of high, medium, and low or no expression (right).

659 Individual colonies analyzed for each transformation event summed from several

660 plates are indicated for each strain. $C$. reinhardtii genetic elements: A - HSP70A

661 promoter, $\mathrm{R}$ - RBCS2 promoter, i1 - RBCS2 intron 1, i2, RBCS2 intron 2, B - beta

662

663

664

665

666

667

668

669

670 tubulin promoter and its 5' untranslated region (UTR), 3'UTR - RBCS2 3' UTR

Figure 2. Buffered phosphite solutions can be used in algal high cell density medium concepts to replace phosphate. A Growth of strain UPN22 was tracked in cultivations in $100 \mathrm{~mL}$ of 6XP (solid line) or 6XPhi (dashed line) media in CellDeg HD100 cultivators following the $\mathrm{CO}_{2}$ and light regime indicated. Spectrum of the Valoya daylight lamp is shown. Cell densities were recorded daily. B Forward and backscatter plots from flow cytometry of samples from day 6 of each culture with photographs of the dense green culture in either medium.

671

672

673

Figure 3: Genetic constructs used to generate heterologous patchoulol production

674

675 from a UPN strain. Single, double, triple, and quadruple copies of the $C$. reinhardtii codon optimized, intron containing $P$. cabiln patchoulol synthase were fused to generate different expression plasmids with C-terminal mVenus (YFP) reporter

676

677 fusions as previously described(Lauersen et al., 2016). Each plasmid was transformed into UPN and mVenus (YFP) expressing colonies were isolated for each construct and benchmarked for patchoulol production $(n=6)$. The chemical structure of patchoulol is shown. The best performing individual from each plasmid was then subsequently transformed with a plasmid expressing a luciferase-amiRNA construct

681 which downregulates the $C$. reinhardtii squalene synthase. Combined high682 throughput fluorescence and luciferase screening of colonies led to isolation of 683 strains with both constructs expressed $(n=6)$ which were then subsequently

684

685 
686 Figure 4. Production of heterologous sesquiterpenoid in the presence of 687 contamination. A C. reinhardtii UPN 22 expressing 2XPCPS-YFP + SQS-amiRNA

688 was cultivated in different trophic modes with and without phosphite and intentionally 689 contaminated with $S$. cerevisiae cells. $\mathrm{TAP}_{-} \mathrm{NO}_{3}$ and $\mathrm{TAPhi}^{-\mathrm{NO}_{3}}$ were used to 690 compare mixotrophic conditions where acetic acid was a sole carbon source, while 691 6xP and 6xPhi were used to test photoautotrophic conditions. All growth curves with 692 Phi are represented with dashed lines and hashed bars. Dodecane overlay was used 693 to capture heterologous patchoulol produced. Yeast cells were intentionally 694 inoculated at high densities to challenge the algal cells to outcompete them in these 695 conditions. $\mathrm{CO}_{2}$ was delivered to autotrophic cultures by placing high-concentration 696 bicarbonate buffer between microtiter plate wells as an inefficient delivery 697 mechanism to further challenge the algal cells. B cultivation of this strain in 6xPhi 698 medium in HD100 cultivator (pictured) with dodecane overlay resulted in efficient 699 patchoulol production from $\mathrm{CO}_{2}$. Two GC-MS chromatograms are shown, one of 700 dodecane blank with alpha-humulene internal standard and one from algal culture 701 indicating the peak of produced patchoulol. 
703

704

705

706

707

708

709

710

711

712

713

714

715

716

717

718

719

720

721

722

723

724

725

726

727

728

729

730

731

732

733

734

735

736

737

738

739

740

741

742

743

744

745

746

747

748

749

750

751

752

753

754

755

\section{Supplemental figure legends}

Supplemental Figure 1: Modifications to the pOpt2 plasmids used in this work. Above: Overview of plasmid architecture of the pOpt 2.0 vectors from Wichmann et al. (2018) 10.1016/j.ymben.2017.12.010. The gene of interest (GOI) reporter cassette is highlighted. Middle: in this work, we modified the GOI cassette to contain the optimized HSP70A, beta-tubulin (and 5'UTR) from Einhaus et al. (2021) 10.1021/acssynbio.0c00632. The mVenus reporter (NCBI: AAZ65844) was also modified to contain two copies of the RBCS2 intron 1, and the RBCS2 intron 2 was moved into the C-terminal Strepll tag, so that future C-terminal fusions could benefit from this orientation in a similar fashion to that presented in Freudenberg et al. (2021) 10.1016/j.biortech.2020.124542. All elements are from C. reinhardtii: A HSP70A promoter, R-RBCS2 promoter, i1 - RBCS2 intron 1, i2, RBCS2 intron 2, B - beta tubulin promoter and its 5' untranslated region (UTR), 3'UTR - RBCS2 3' UTR. AmpR - ampicillin resistance cassette of the pBluescript SK(+) backbone. Below: Plasmids for the expression of the $C$. reinhardtii codon optimized and synthetic intron-containing Pogostemon cablin patchoulol synthase (UniProt: Q49SP3, PcPS). Modified pOpt2.0 expression cassettes were used to subclone the PCPS which had been previously codon optimized (including intron spreading) for expression from the nuclear genome of $C$. reinhardtii (NCBI: KX097887, Lauersen et al. (2016) 10.1016/j.ymben.2016.07.013). All plasmids have the pOpt2.0 paromomycin resistance cassette $(\mathrm{P}) 3^{\prime}$ ' of the $\mathrm{GOI}$ expression cassette pictured (as in Wichmann et al. (2018)). Subcloning of PcPS plasmid 1X was performed in the lab of Prof. Dr. Olaf Kruse by Dr. Julian Wichmann and Dr. Thomas Baier as part of an Institute for Innovation Transfer (IIT) project funded by Lauersen (KAUST). Further subcloning of $2 \mathrm{X}, 3 \mathrm{X}, 4 \mathrm{X}$ were performed by Dr. Gordon Wellman in Lauersen's lab at KAUST. Abbreviations: $Y$ - mVenus expression cassette, 1X, 2X, 3X, 4X plasmids contain the respective numbers of PCPs copies. Cloning was achieved by using the previous plasmid, with Scal-BamHI as the receiving vector and Scal-Bgll as an insert to amplify the PcPS coding sequence.

Supplemental Figure 2: Confirmation of pPO3 integration into the chloroplast genome of UVM4 in derivative strains UVM4-phi and UPN22. Primers Fw: AATTGTATGGGCTCACAACAAACTTAAAGT and Rv: TAAAATTGTGAGACCATGAGTAATGTTCCTCC were used to perform PCR on DNA extracts from each strain. The target region without amplification should yield $1050 \mathrm{bp}$ band, while integration should yield 3075 bp products.

Supplemental Figure 3: In gel fluorescence of SDS PAGE samples from one representative mutant of each of the genetic constructs indicated. Fluorescence image was captured with 510/10 nm excitation and 530/10 nm emission filter in the Analytik Jena Chemstudio Plus with eLite. White contrast black and white image was taken without emission filter using 510/10 nm excitation to visualize the marker.

Supplemental Figure 4: Patchoulol productivities observed in dodecane overlays for 6 transformants selected for bright YFP fluorescence from each of the above plasmids and compared to parental UPN strain and empty vector $(\mathrm{Y})$ generated control strains. Numbers at the bottom of the graph correspond to plasmid name and mutant number $(1.1=1 \mathrm{X}$ PCPS, transformant \#1). Productivity-grouped averages are shown on the left and labelled with each plasmid name.

Supplemental Figure 5: Patchoulol productivities observed in dodecane overlays for six transformants isolated from secondary transformation of the best 1-4X PCPS 
756 strains with CCA_gLuc_i3_SQSk.d. plasmid (Wichmann et al. 2018). Upper right:

757 representitive GC-MS chromatograms showing the drastic increase of patchoulol

758 production in SQS k.d. secondary transformants relative to alpha-humulene internal

759 standard. Lower graphs: Numbers at the bottom of the graph correspond to plasmid

760 name and mutant number (1x1 = 1X PCPS SQS k.d. mutant 1). The averages of all

761 transformants per group are shown on the left and labelled with each plasmid name.

762 Corresponding parent performances are also shown from the data in previous figure.

763

764

765 
bioRxiv preprint doi: https://doi.org/10.1101/2022.02.28.482248; this version posted March 1, 2022. The copyright holder for this preprint (which was not certified by peer review) is the author/funder, who has granted bioRxiv a license to display the preprint in perpetuity. It is made available under aCC-BY-NC-ND 4.0 International license.

\section{Tables}

767 Supplemental Table 1. Genetic constructs used in this study. Plasmids for

768 transformation of $C$. reinhardtii are shown as well as some of their respective 769 properties. References to plasmid sequences are given and those generated in this 770 work are provided in the supplement. 


\section{5. Contribution to the field statement}

774 In this work, we combine previously published optimizations in algal metabolism in a 775 mutant workhorse for nuclear transgene expression. We expand this strain with the 776 metabolic capacity for use of inorganic phosphite as a sole phosphorous source, and 777 nitrate as a nitrogen source. These combined engineering steps enable the 778 cultivation of this strain in both high-density culture media, with loading of nitrates to 779 increase cell densities, and the use of phosphite to mitigate contamination. We 780 present recipes for replacing phosphate buffers with phosphite buffers and 781 demonstrate heterologous production of the sesquiterpenoid patchoulol by 782 combining synthase overexpression with competitive pathway knockdown. Our work 783 is the first example of combinatorial chloroplast and nuclear genome engineering for 784 heterologous metabolite engineering in a green algal host and sets an example for 785 future engineering in these hosts to enable scalable cultivation concepts. 

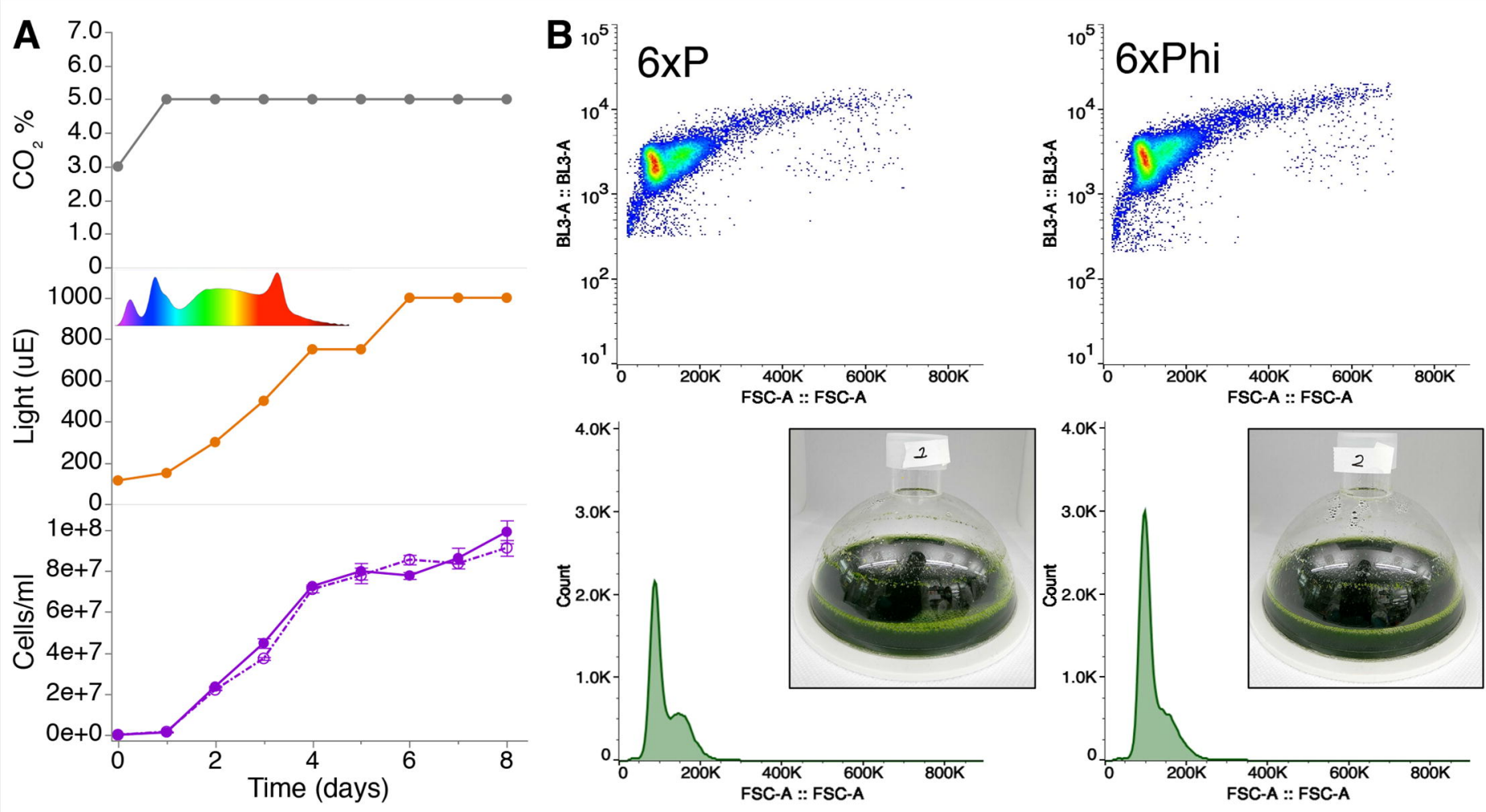


\section{$\Rightarrow \mathrm{pHSP70A} \mathbf{P}$ ParomomycinR}

$\Rightarrow$ pRBCS2

$\Rightarrow$ pßtubulin $\mathbf{S}$ SpectinomycinR

$\square \square \square P C P S \square \square \mathbb{l l l}$ gLuc-SQS

$\square \square \square$ mVenus

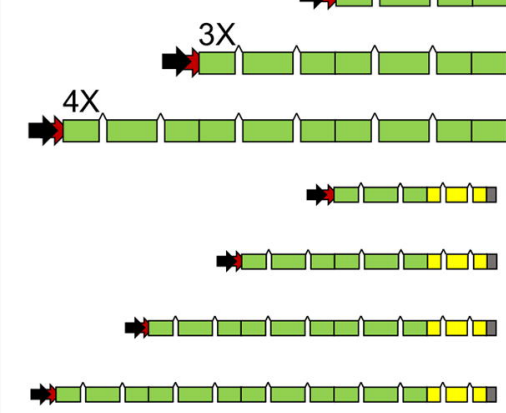

Patchoulol (fg cell $\left.{ }^{-1}\right)$ 
$\mathbf{A}_{1.6 \mathrm{e}+7}$

TAP $\mathrm{NO}_{3}$

$\mathrm{TAPhi} \mathrm{NO}_{3}$

$6 \mathrm{xP}+\mathrm{CO}_{2}$

$6 \times \mathrm{Phi}+\mathrm{CO}_{2}$
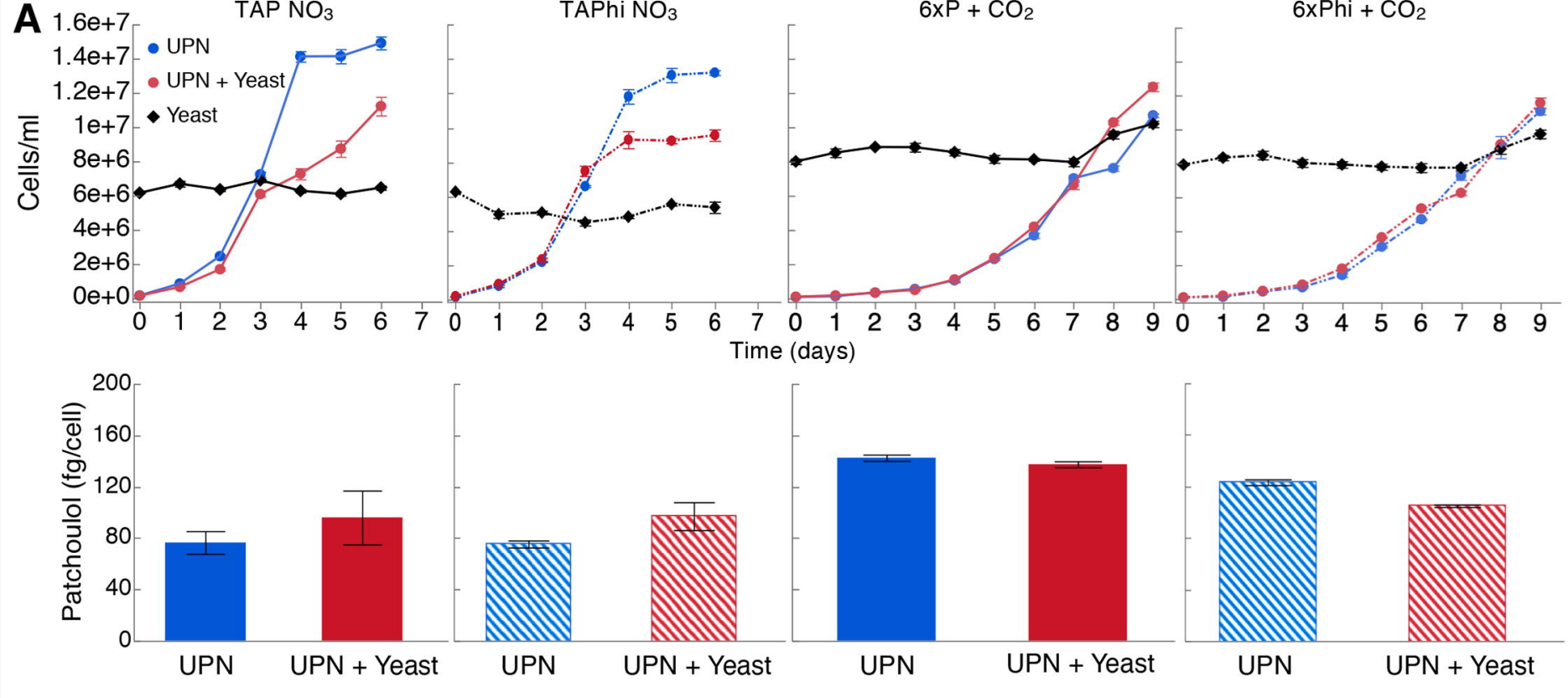

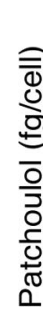
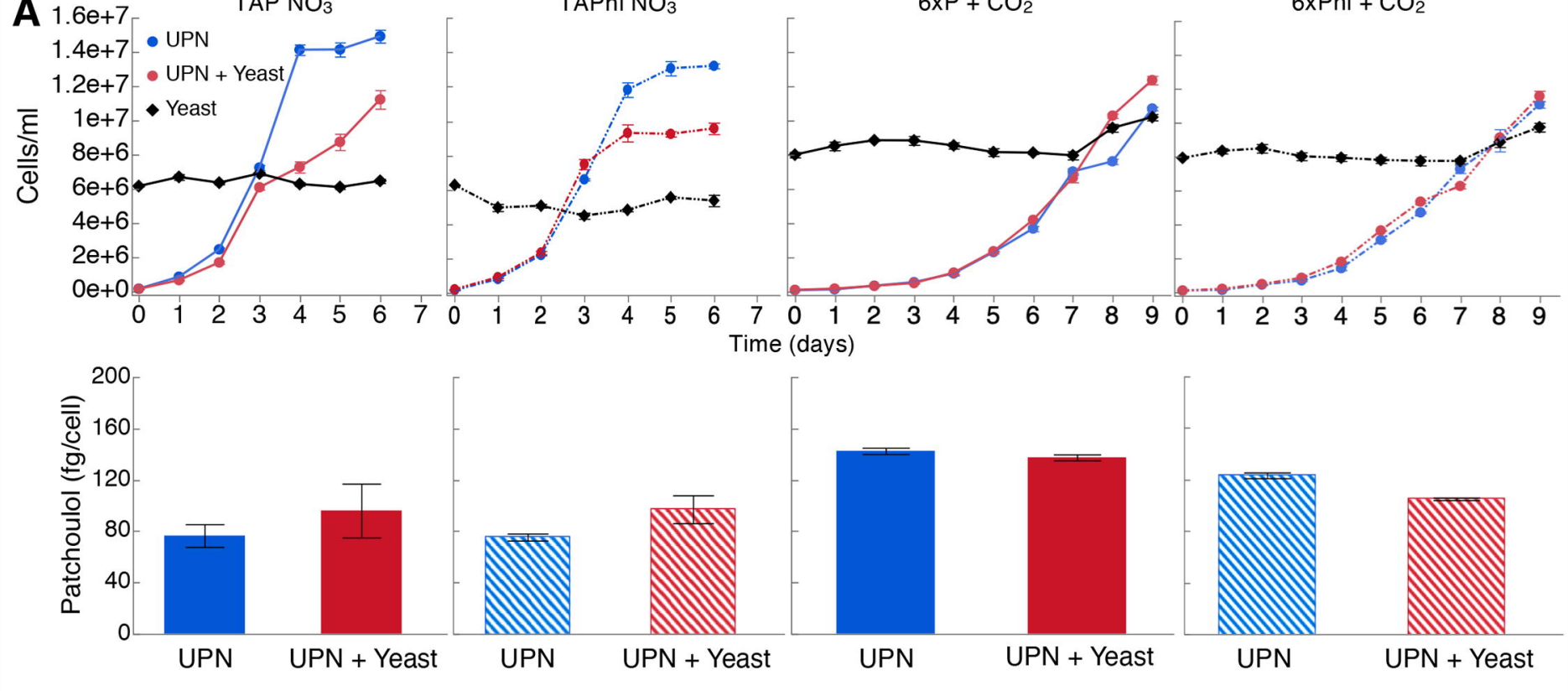

UPN + Yeast

B
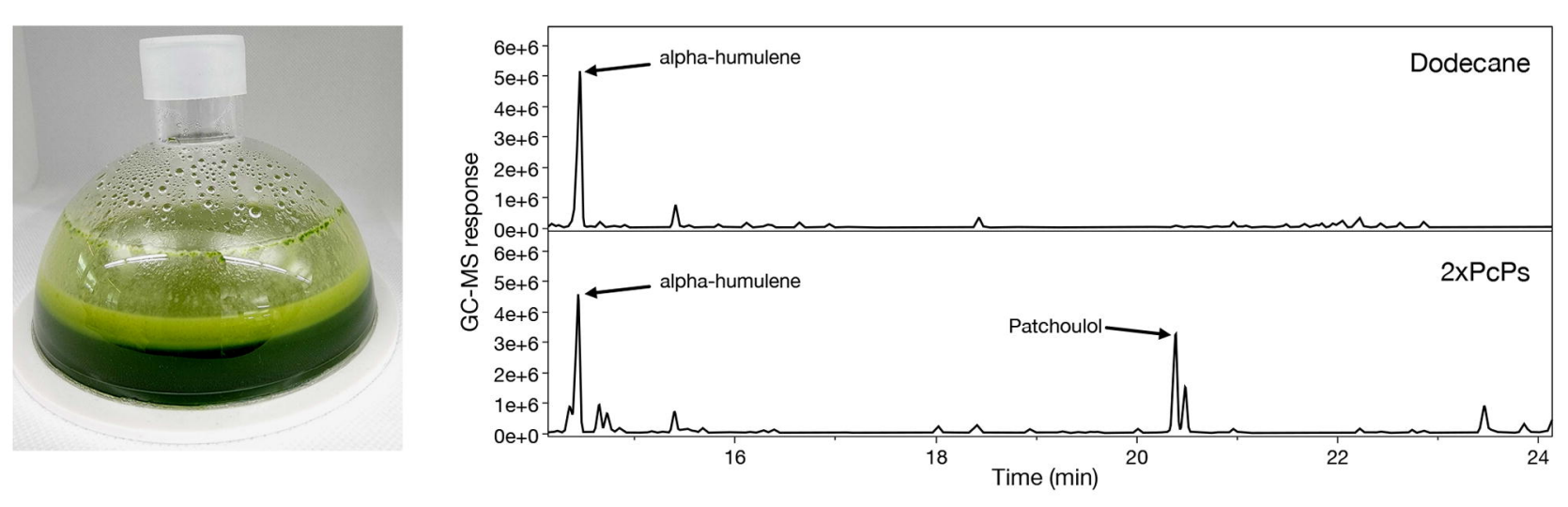

B 